\title{
The Difference in Cinical and Prognostic Features Between De Novo and Recurrent Her2-Positive Metastatic Breast Cancer Patients
}

\author{
Yusuf Acikgoz ${ }^{1, *},[M D]$ \\ ORCID:0000-0002-0360-7938 \\ Yakup Ergun',[MD] \\ ORCID: 0000-0003-4784-6743 \\ Gökhan Ucar ${ }^{1}$, [MD] \\ ORCID:0000-0002-7649-1075 \\ Merve Dirikoc ${ }^{1},[\mathrm{MD}]$ \\ ORCID:0000-0002-3762-7152 \\ Dogan Uncu', [MD] \\ ORCID:0000-0002-0929-3271
}

${ }^{1}$ Ankara Numune Education and Research Hospital
Department of Medical Oncology

Corresponding Author: Yusuf Açıkgöz, MD ORCID ID: 0000-0002-0360-7938

Ankara Numune Education and Research Hospital, Department of Medical Oncology

06100, Ankara, TURKEY

Phone: +903125084603 , Fax:+903125084914

E-mail:yacikgoz86@gmail.com

\section{wee ABSTRACT Cen}

Objective: There are different data in the literature about the consequences of the development of metastasis as de novo or recurrent. In this study, we retrospectively investigated the clinicopathologic and prognostic characteristics of HER-2 positive de novo and recurrent metastatic breast cancer patients.

Material and Methods: The data of patients admitted to our clinic between 19962017 were analysed retrospectively. The baseline features, treatments and survival data were recorded. Recurrent metastatic patients were further categorized as disease free interval $<24$ months and disease free interval $>24$ months. The features of two groups were analysed by Pearson chi-square test. Survival were calculated by using the Kaplan-Meier method with the Long-rank test. $\mathrm{p}<0.05$ was considered statistically significant.

Results: A total of 44 patients were included to study in which 20 patients in de novo HER-2 positive metastatic breast cancer group and 24 patients in recurrent HER-2 metastatic breast cancer group. There was no difference in baseline features between groups. The median OS in de novo and recurrent $\mathrm{MBC}$ group was 60.3 months and 43.9 months respectively (HR: $0.87,95 \% \mathrm{CI} 0.37-2.05, \mathrm{p}=0.76$ ). OS was not different between de novo metastatic breast cancer group and patients with disease free inter$\mathrm{val}<24$ months and with disease free interval $>24$ months $(\mathrm{p}=0.135)$.

Conclusion: Our study showed that baseline features of patients with de novo HER2 positive $\mathrm{MBC}$ and recurrent HER-2 positive metastatic breast cancer did not differ from each other. The presence of metastasis at the time of diagnosis or during follow-up did not change response to treatments.

Key words: Breast cancer, HER-2 positive, metastatic, de novo, recurrent

Received: 04 October 2019, Accepted: 04 December 2019,

Published online: 31 December 2019

\section{INTRODUCTION}

Breast cancer is the most diagnosed malignancy and one of the leading cause of death among women. In 2018, it is estimated that 266.120 new cases and 40.920 deaths will occur in USA and $6 \%$ of those is predicted to be at stage IV. Despite modern treatments, the expected 5-year survival rate in patients with stage IV breast cancer is still approximately $27 \%[1,2]$.

Metastatic breast cancer is composed of two different groups as de novo metastatic breast cancer (MBC) and recurrent metastatic breast cancer depending on the time of metastasis detected [3]. De novo and recurrent $M B C$ show some differences in terms of treatment response and prognosis [4]. Similar to other tumours, some factors such as age, performance status, and distant organ metastasis effect the prognosis and outcomes [5]. In a large study conducted on metastatic breast cancer in the period between 1992-2007 showed that patients with de novo MBC have better outcomes compared with recurrent $M B C$. Further analysis of this trial showed that interval between diagnosis and metastasis is an important parameter that may be related to this different outcomes [6]. This better prognosis has also been observed in the de novo human 
epidermal growth factor receptor 2 (HER-2) positive MBC subgroup.

There are number of hypotheses which aim to explain the prognostic difference between de novo and recurrent MBC. Of those, the widely accepted one is that patients with recurrent $M B C$ become more resistant to chemotherapeutics due to previous treatment that they had received [7]. Until now; the factors accurately define these differences has not been very well known. Conversely, Lobbezoo DJ et al showed that the survival difference between that two groups did not change noticeably; even after the exclusion of patients with recurrent MBC who had previously received certain treatments [8]. In this study, we aimed to demonstrate differences or similarities between these two groups and additionally to identify factors that may give rise to differences with regards to survival and treatment response between patients with de novo and recurrent HER-2 positive MBC.

\section{MATERIALS and METHODS}

\section{Study Populations}

This retrospective study was approved by institutional ethics committee of Ankara Numune Training and Research Hospital. We retrospectively reviewed clinical records of de novo and recurrent HER-2 positive $M B C$ followed in our centre between date June 1996 and November 2017. A total of 44 patients' data were reached and pooled from oncologic follow-up files in our centre. Eligibility criteria were determined as following; histologically confirmed HER-2 positive breast cancer, radiologically or histologically confirmed metastasis either at the time of presentation or during follow-up and being over age 18. Patients who had second primary cancer, non-metastatic disease, and male gender excluded from the study.

Various factors were recorded including age at the time of diagnosis and metastases, site of metastases, hormone receptor status, HER-2 status, histological subtypes and grade, progression-free survival (PFS), overall survival (OS) and disease free interval for recurrent patients. Disease free interval (DFI) was defined as the time between diagnosis of nonmetastatic breast cancer and the development of distant recurrence. Progression-free survival was determined as the time between the beginning of treatment and first progression or death from any cause, whichever occurred first. Patients with recurrent $M B C$ were classified in similar manner to the studies which conducted before as having $\mathrm{DFI}<2$ years or $>2$ years $[4,8]$. Data about patients' demographic characteristics, survival status, treatment protocols, sites and number of metastasis yielded from oncologic follow-up files. In addition, we specifically recorded brain metastasis under a different category as having brain metastasis or not having.

\section{Staging and Pathology}

The disease was staged according to the TNM staging in the $7^{\text {th }}$ edition of American Joint Committee on Cancer Staging Manual [9]. Histological features of tumour including hormone receptor status, HER2 score and histological grade were obtained from pathology reports. The hormone receptor status, for both estrogen and progesterone, was recorded as percentage in numerical form. Patients whose tumour with immunohistochemical staining score was $3+$, or $2+$ with a positive fluorescence in situ hybridization considered as HER2 positive disease.

\section{Statistical Analysis}

Statistical analyses were performed by using Statistical Package for the Social Sciences Version 22.0 for Windows (SPSS Inc., Chicago, IL, USA). We categorized patients into two main groups as de novo or recurrent breast cancer. Baseline features of two groups compared by Pearson chi-square test and Mann-Whitney $U$ test, as appropriate. We used Kaplan-Meier test for survival, and outcomes were analysed by Log-rank test. Overall survival was calculated from the date of first distant metastasis to date of last control for alive patients or death from any cause. PFS was calculated from the date of beginning treatment in metastatic setting to the date of death or progression, whichever occurred first. A $p$-value $<0.05$ considered statistically significant.

\section{RESULTS}

A total of forty-four patients with HER-2 positive metastatic breast cancer were included in this study. The number of patients in de novo HER-2 positive MBC group was twenty (45.5\%) and twenty-four (54.5\%) in recurrent HER-2 positive MBC group. The median age of the whole group was 47 (range; 30-71) and the number of premenopausal patients was 34 (77.3\%). Most of the patients had ECOG performance score $0-1$ with the number of 38 (86.4\%). There were no statistically significant differences between two groups in terms of baseline characteristics. Table- 1 provides the information 
Her2-positive metastatic breast cancer patients

about patient and tumour characteristics categorized by de novo HER-2 positive $M B C$ and recurrent HER-2 positive MBC patients. 15 (65.2\%) patients with recurrent HER-2 positive MBC had received trastuzumab in adjuvant or neoadjuvant settings. The ratio of patients who received at least one-line chemotherapy was $95 \%$; $61 \%$ of patients received at least two lines chemotherapy and $38 \%$ of patients received three or more lines of chemotherapy in metastatic settings. When the treatment of patients in the first line was examined, the number of patients who received trastuzumab-based treatments was $16(80 \%)$ vs $10(41.6 \%)$ in de novo MBC and recurrent $M B C$ group respectively (Table-2). All patients in entire cohort could be assessed for response; 3 patients $(6.8 \%)$ had complete response (CR), 15 patients (34.1\%) had partial response (PR), 14 patients (31.8\%) had stable disease (SD) and 12 patients (27.3\%) had progressive disease. Objective response rate was $47.4 \%$ and $42.9 \%$ for de novo HER-2 positive $M B C$ and recurrent HER-2 positive MBC respectively $(p=0.775)$.

Table 1. Patient and tumour characteristics categorized by de novo HER2-positive MBC vs recurrent HER-2 positive MBC

\begin{tabular}{|c|c|c|c|}
\hline Characteristics & $\begin{array}{c}\text { De novo } \\
\text { MBC } \\
\text { n (\%) }\end{array}$ & $\begin{array}{c}\text { Recurrent } \\
\text { MBC } \\
\text { n (\%) }\end{array}$ & P value* \\
\hline Number of patients & $20(45.5)$ & $24(54.5)$ & 0.36 \\
\hline $\begin{array}{l}\text { Median age at diagnosis, } \\
\text { years (range) }\end{array}$ & $50(32-71)$ & $47(30-61)$ & 0.22 \\
\hline \multicolumn{4}{|l|}{ Menopausal status } \\
\hline Premenopausal & $14(70)$ & $20(83.3)$ & 0.47 \\
\hline Postmenopausal & $6(30)$ & 4 (16.7) & \\
\hline \multicolumn{4}{|l|}{$\begin{array}{l}\text { ECOG performance status at } \\
\text { time of metastasis }\end{array}$} \\
\hline $0-1$ & $17(85)$ & $21(87.5)$ & 1.00 \\
\hline$\geq 2$ & $3(15)$ & $3(12.5)$ & \\
\hline \multicolumn{4}{|l|}{ Hormone receptor status } \\
\hline ER or PR positive & $15(75)$ & $17(70.8)$ & 0.74 \\
\hline ER and PR negative & $5(25$ & $7(29.2)$ & \\
\hline \multicolumn{4}{|l|}{ Histology } \\
\hline Invasive ductal & $19(95)$ & $23(95.8)$ & 1.00 \\
\hline Other & $1(5)$ & $1(4.2)$ & \\
\hline \multicolumn{4}{|l|}{ Histological Grade } \\
\hline । & $5(25)$ & $6(25)$ & 0.73 \\
\hline ॥ & $8(40)$ & $12(50)$ & \\
\hline III & $7(35$ & $6(25)$ & \\
\hline \multicolumn{4}{|l|}{ Brain metastasis } \\
\hline Positive & $5(25)$ & $6(25)$ & 1.00 \\
\hline Negative & $15(75$ & $18(75)$ & \\
\hline
\end{tabular}

*Comparison of two groups was performed by Pearson chi-square test and Mann-Whitney U test. 
Table 2. First line treatment for MBC

\begin{tabular}{|l|c|c|}
\hline First Line Treatments & $\begin{array}{c}\text { De novo } \\
\text { MBC } \\
\mathrm{n}(\%)\end{array}$ & $\begin{array}{c}\text { Recurrent } \\
\mathrm{n}(\%)\end{array}$ \\
\hline Number of patients & $20(45.5)$ & $24(54.5)$ \\
\hline Trastuzumab-based treatments & & $10(41.6)$ \\
With Taxanes & $16(80)$ & $3(12.5)$ \\
With Vinorelbine & 0 & $1(4.1)$ \\
Single agent & $1(5)$ & $3(12.5)$ \\
\hline Non-trastuzumab treatments & $3(15)$ & $7(29.3)$ \\
AC & 0 & \\
Capecitabine or Platinum & & \\
\hline
\end{tabular}

The median follow-up time was 32.7 months (1.2-112) for whole group, while it was 37.9 months (1.2-92.4) and 25.9 months (3.1-112) for de novo HER-2 positive MBC and recurrent HER-2 positive MBC patients, respectively. Survival analysis of the whole group showed that total number of death was 22 (50\%); 11 (55.0\%) in de novo HER-2 positive MBC group and $11(45.8 \%)$ in recurrent HER-2 positive MBC group. Overall survival was 52.4 months $(95 \% \mathrm{Cl} 38.6-66.2)$ for whole groups while it was 60.3 months (95\% Cl 27.7-92.9) for de novo HER-2 positive MBC and 43.9 months $(95 \% \mathrm{Cl} 30.3-57.5)$ for recurrent HER-2 positive MBC. Overall survival was numerically longer in de novo HER-2 positive $M B C$ but it did not reach a statistical significance (Figure-1) $(p=0.76)$. The median PFS for de novo MBC patients was 16.8 months $(95 \% \mathrm{Cl} 0.6-32.9)$ and 10.7 months $(95 \% \mathrm{Cl} 6.2-15.1)$ for recurrent MBC patients $(p=0.68)$.

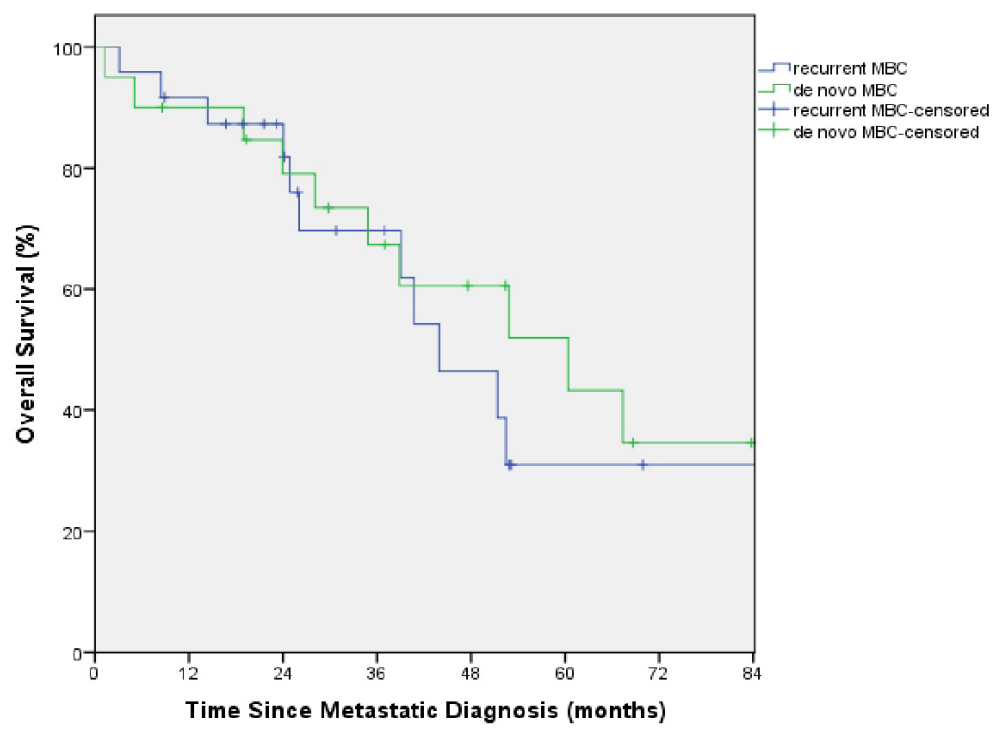

Figure 1. Overall survival for de novo $M B C$ and recurrent $M B C$

Overall survival data of patients with recurrent HER-2 positive MBC stratified regarding to DFI as relapsing before 24 months and after 24 months. Overall survival of recurrent HER-2 positive MBC patients with DFI $<24(n=6)$ was 26.0 months $(95 \% \mathrm{Cl} 22.9-29.1)$ and for patients with DFI $>24(n=18)$ was 52.4 months $(95 \% \mathrm{Cl} 35.1-69.7)(\mathrm{p}=0.065)$. As it is shown in Figure-2, there was no statistically significant difference in OS between de novo HER-2 positive MBC group and patients with $\mathrm{DFI}<24$ months and patients with $\mathrm{DFI}>24$ $(p=0.135)$. 


\section{DISCUSSION}

In our study, we aimed to demonstrate whether there is any difference in prognostic outcomes and clinicopathologic features between de novo HER2 positive MBC and recurrent HER-2 positive MBC patients. We observed that the median overall survival of patients with de novo HER-2 positive MBC was 16.4 months longer than that of recurrent HER2 positive $\mathrm{MBC}$ which has not reached statistical significance. Additionally, there were no differences in terms of PFS and baseline clinicopathological features. We could not find any statistically difference with regard to age, menopausal status, histological features of tumors and ECOG performance status at the time of metastasis.

There is no doubt that survival of patients' metastatic breast cancer is increasing in past decades. There are several studies that showed this improvement in patients with metastatic breast cancer is irrespective of de novo or recurrence status $[10,12]$. This trend most probably related to newer therapeutic agents [12]. There are some studies that showed patients with de novo MBC have superior outcomes compared with recurrent $\mathrm{MBC}[6,8,13]$. Dawood et al, reported better outcomes of survival and lower death risk for de novo MBC over recurrent $M B C$ patients from the study which was conducted with 3524 patients, in which 563 patients had HER-2 positive MBC, at The University of Texas M.D. Anderson Cancer Centre [6]. Unlike the results of our study, registHER study which was conducted as a prospective observational study with 1023 patients showed that patients with de novo HER2 positive $\mathrm{MBC}$ had longer OS and PFS comparing to the patients with recurrent HER-2 positive MBC. However, in adjuvant or neoadjuvant settings, only 55 of 674 patients had received HER-2 targeted therapy in recurrent HER-2 positive MBC group owing to timing of this study [7]. It is a well-known fact that anti HER-2 therapy is very effective in reducing the risk of recurrence [14-16]. Thereby, this favourable outcome of de novo HER-2 positive MBC group may be related to limited using of anti HER2 therapy. In another study which was conducted by V.Rossi et al showed similar to the results of our study. A sum of 331 patients included in this study, of those 77 (23\%) patients had de novo HER-2 positive MBC and 254 (77\%) had recurrent HER-2 positive MBC. In this study, OS and PFS did not show any differences with respect to their recurrence status
[17]. Similarly, we did not find statistically significant difference in OS for both groups in our study.

On the other hand, data from a variety studies showed that survival among recurrent MBC differed with regard to DFI. According to these studies, survival of patients with $\mathrm{DFl}<2$ years was poorer than patients with $D F \mid>2$ years. Survival of patients with $D F l>2$ years was almost equal to that of de novo MBC patients $[4,8]$. In contrast to these studies, we could not find any statistically significant difference in OS between the groups. This partly may be due to low number of patients in each group.

Our study has limitations due to its retrospective nature, relatively small patients number and timing of study. Some patients had been diagnosed with HER2 positive MBC before era of trastuzumab. Hereby, 9 (39.1\%) patients did not receive trastuzumab in adjuvant or neoadjuvant settings which is not compatible with our updated clinical practice. However, absence of trastuzumab in adjuvant or neoadjuvant setting did not caused any differences for OS in the CLEOPATRA trial in which only $10 \%$ of patients had received trastuzumab in adjuvant or neoadjuvant setting [18]. Despite this similarity, we still think that absence of trastuzumab in adjuvant or neoadjuvant settings probably caused biases in OS analysis through giving rise to early recurrence.

In conclusion, metastatic breast cancer has been generally considered as a uniform entity. Although little research has been done in this area, a limited number of study showed the difference between de novo and recurrence MBC in terms of OS, PFS and clinicopathological features. Our study did not show any differences between de novo HER-2 positive MBC and recurrent HER-2 positive MBC. Some patients had received treatment before era of trastuzumab which can remarkably effect survival and other outcomes. Due to limited data about these two different entities, further analysis which includes features of tumour biology should be conducted.

\section{ACKNOWLEDGEMENT}

The study protocol has been approved by the research institute's committee on human research.

\section{CONFUCTS of INTEREST}

The authors have no conflicts of interest to declare 
[1] National Cancer Institute. Surveillance, Epidemiology, and Endb Results Program. SEER Stat Fact Sheets: Breast Cancer. https://seer.cancer.gov/statfacts/html/breast.html. Published 2018. Accessed.

[2] National Cancer Institute. Surveillance, Epidemiology, and End Results Program. SEER Stat Fact Sheets: Common Cancer Sites. https://seer.cancer.gov/statfacts/html/common.html. Published 2018. Accessed.

[3] Engel-Nitz NM, Hao Y, Becker LK, et al. Costs and mortality of recurrent versus de novo hormone receptor-positive/HER2(-) metastatic breast cancer. Journal of Comparative Effectiveness Research 2015; 4(4): 303-14.

[4] Yamamura JUN, Kamigaki S, Fujita J, et al. The Difference in Prognostic Outcomes Between De Novo Stage IV and Recurrent Metastatic Patients with Hormone Receptor-positive, HER2negative Breast Cancer. In Vivo 2018; 32(2): 353-58.

[5] Yamamoto N, Watanabe T, Katsumata N, et al. Construction and validation of a practical prognostic index for patients with metastatic breast cancer. Journal of clinical oncology: official journal of the American Society of Clinical Oncology 1998; 16(7): 2401-08.

[6] Dawood S, Broglio K, Ensor J, et al. Survival differences among women with de novo stage IV and relapsed breast cancer. Annals of oncology: official journal of the European Society for Medical Oncology 2010; 21(11): 2169-74.

[7] Yardley DA, Kaufman PA, Brufsky A, et al. Treatment patterns and clinical outcomes for patients with de novo versus recurrent HER2-positive metastatic breast cancer. Breast Cancer Research and Treatment 2014; 145(3): 725-34.

[8] Lobbezoo DJ, van Kampen RJ, Voogd AC, et al. Prognosis of metastatic breast cancer: are there differences between patients with de novo and recurrent metastatic breast cancer? British Journal of Cancer 2015; 112(9): 1445-51.

[9] Edge SB, Compton CC. The American Joint Committee on Cancer: the 7th Edition of the AJCC Cancer Staging Manual and the Future of TNM. Annals of Surgical Oncology 2010; 17(6): 1471-74.
[10] Dawood S, Broglio K, Gonzalez-Angulo AM, et al. Trends in survival over the past two decades among white and black patients with newly diagnosed stage IV breast cancer. Journal of clinical oncology: official journal of the American Society of Clinical Oncology 2008; 26(30): 4891-98.

[11] Giordano SH, Buzdar AU, Smith TL, et al. Is breast cancer survival improving? Cancer 2004; 100(1): 44-52.

[12] Andre F, Slimane K, Bachelot T, et al. Breast cancer with synchronous metastases: trends in survival during a 14-year period. Journal of clinical oncology: official journal of the American Society of Clinical Oncology. 2004; 22(16): 3302-08.

[13] den Brok WD, Speers CH, Gondara L, et al. Survival with metastatic breast cancer based on initial presentation, de novo versus relapsed. Breast Cancer Research and Treatment 2017; 161(3): 549-56.

[14] Piccart-Gebhart MJ, Procter M, Leyland-Jones B, et al. Trastuzumab after adjuvant chemotherapy in HER2-positive breast cancer. The New England Journal of Medicine 2005; 353(16): 1659-72.

[15] Romond EH, Perez EA, Bryant J, et al. Trastuzumab plus adjuvant chemotherapy for operable HER2-positive breast cancer. The New England Journal of Medicine 2005; 353(16): 1673-84.

[16] Slamon D, Eiermann W, Robert N, et al. Adjuvant trastuzumab in HER2-positive breast cancer. The New England Journal of Medicine 2011; 365(14): 1273-83.

[17] Rossi V, Nole F, Redana S, et al. Clinical outcome in women with HER2-positive de novo or recurring stage IV breast cancer receiving trastuzumab-based therapy. Breast 2014; 23(1): 44-49. [18] Swain SM, Kim S-B, Cortés J, et al. Pertuzumab, trastuzum$\mathrm{ab}$, and docetaxel for HER2-positive metastatic breast cancer (CLEOPATRA study): overall survival results from a randomised, double-blind, placebo-controlled, phase 3 study. The Lancet Oncology 2013; 14(6): 461-71. 